\title{
Stochastic approach to gravitational waves from inflation
}

\author{
Gianmassimo Tasinato \\ Physics Department, Swansea University, SA28PP, United Kingdom
}

\begin{abstract}
We propose a coarse-graining procedure for describing the superhorizon dynamics of inflationary tensor modes. Our aim is to formulate a stochastic description for the statistics of spin-2 modes which seed the background of gravitational waves from inflation. Using basic principles of quantum mechanics, we determine a probability density for coarse-grained tensor fields, which satisfies a stochastic Fokker-Planck equation at superhorizon scales. The corresponding noise and drift are computable, and depend on the cosmological system under consideration. Our general formulas are applied to a variety of cosmological scenarios, also considering cases seldom considered in the context of stochastic inflation, and which are important for their observational consequences. We start obtaining the expected expressions for noise and drift in pure de Sitter and power-law inflation, also including a discussion of effects of non-attractor phases. We then apply our methods to describe scenarios with a transition from inflation to standard cosmological eras of radiation and matter domination. We show how the interference between modes flowing through the cosmological horizon, and modes spontaneously produced at superhorizon scales, can affect the stochastic evolution of coarse-grained tensor quantities. In appropriate limits, we find that the corresponding spectrum of tensor modes at horizon crossing matches with the results of quantum field theory calculations, but we also highlight where differences can arise.
\end{abstract}




\section{Introduction}

Cosmological inflation is the most successful mechanism at our disposal for generating the initial conditions for our universe [1-7]. During cosmological inflation, space-time fluctuations produced by quantum effects at microscopic distances are stretched to superhorizon scales, where they freeze. Subsequently, after inflation ends, such large-scale fluctuations reenter the horizon, they become dynamical, and seed the evolving cosmic structures we observe today in the sky.

This picture of early universe cosmology is appealing and physically well motivated. Nevertheless, potentially large infrared effects require specific care when applying pertubative quantum field theory techniques to cosmology. For example, it is well known [8-10] that perturbative computations of correlation functions of light quantum fields in de Sitter space can be affected by infrared contributions, making subtle a proper physical interpretation of the calculations: see e.g. [11-16]. A promising proposal to deal with these issues is the stochastic approach to cosmological inflation first proposed by Starobinsky [17], which provides a consistent framework for resumming large infrared effects in de Sitter space: see e.g. [18-31]. The starting point of stochastic inflation is the observation that after crossing the cosmological horizon, quantum fluctuations classicalize [32-40], and their description is more conveniently formulated in terms of a classical, stochastic Fokker-Planck evolution equation. In this perspective, long wavelength modes at superhorizon scales receive impulses from small-scale fluctuations as the latter cross the horizon, a process that intuitively corresponds to a cosmological version of Brownian motion. The resulting stochastic cosmological equations can be handled consistently, and provide information on the global dynamics of the system at the largest cosmological scales, which is difficult to gain otherwise.

In this work we study the stochastic distribution of cosmological fluctuations at superhorizon scales, focusing on the dynamics of primordial tensor modes predicted by inflation [41-44]. Spin-2 inflationary tensor modes are light fields in quasi-de Sitter space: their superhorizon distribution is likely to be amenable of a classical description as in the stochastic approach to scalar fluctuations during cosmological inflation. Specifically, we aim to address two questions:

- Question 1: Is there a way to define coarse-grained tensor modes at superhorizon scales, and study their corresponding dynamics using a stochastic approach?

A reliable stochastic formalism applied to inflationary spin-2 fields would allow us to discuss the dynamics of superhorizon tensor modes using statistical methods, without having to make specific assumptions on the behavior of the individual modes after they cross the horizon during inflation.

- Question 2: Is there a consistent stochastic description for superhorizon inflationary tensor modes after the end of inflation?

This issue has important implications for cosmology, since after inflation ends tensor modes reenter the horizon forming the stochastic background of primordial gravitational waves currently searched by dedicated experiments. Their properties depend on the amplitude and properties of the spectrum at horizon crossing, which depend on the stochastic distribution of tensor modes at the largest, superhorizon scales. 
The answer to Question 1 requires a definition of tensor 'zero-modes' which can be subtle since superhorizon spin-2 fields do not preserve the isotropy of the underlying Friedmann-RobertsonWalker (FRW) space-time. Starting from basic principles of quantum mechanics, in section 2 we propose a coarse-grained description of primordial tensor modes, based on the method of the functional Schrödinger picture [45] used in [27,29] for a stochastic analysis of the scalar sector of fluctuations. The coarse-grained tensor quantity we define is representative of the dynamics of long wavelength tensor modes once they leave the cosmological horizon. Its definition does not interfere with the symmetries of the background space-time. In fact, we focus on a free theory described by a quadratic tensor action, with the specific purpose of understanding how the properties of the coarse-grained quantities depend on the curved cosmological space-time where they are embedded. We obtain a probability density for the coarse-grained superhorizon tensor modes, and we derive its corresponding classical Fokker-Planck evolution equation. It is built in terms of noise and drift, which are explicitly calculable from combinations of mode functions evaluated at superhorizon scales. The definitions of noise and drift are free from large infrared effects. The noise is induced by a flow of modes as they cross the cosmological horizon from small to large scales (or vice versa) - as in the aforementioned cosmological analog of Brownian motion. But it can also be affected by phenomena occurring beyond horizon crossing scales, as for example interference among the flow of modes with particles produced at superhorizon scales by sizable space-time gradients.

The evolution of the coarse-grained probability density is Markovian, up to contributions associated with modes that rapidly decay at superhorizon scales. Such effects make the structure of evolution equations dependent on initial conditions, but they are negligible in scenarios where cosmological evolution is an attractor. However, they can provide a sizable contribution to the drift term in scenarios that include phases of non-attractor evolution, and our general formulas can be applied to those set ups as well.

We also derive formulas for the spectrum of tensor fluctuations evaluated at horizon crossing, which is useful for comparing with results from QFT computations. Moreover, since we are dealing with coarse-grained quantities, we can define a Gibbs entropy for the system at superhorizon scales. We find that it increases with the universe expansion, and we quantitatively characterize its growth.

As far as we are aware, we are the first in attempting to answer Question 2 in the context of a stochastic description of superhorizon tensor modes. We start in section 3.1 with the case of inflation: we recover the expected results for the stochastic distribution of coarse-grained tensor fields during de Sitter and power-law cosmological expansion. We also consider the case for non-attractor cosmological evolution, showing explicitly how it affects the drift contributing to the Fokker-Planck equation. In section 3.2 we apply our stochastic formulas to the case of radiation and matter dominated eras occurring after inflation ends. These stochastic equations describe the coarse-grained evolution of superhorizon tensor modes that eventually reenter the horizon as cosmic evolution proceeds. The computation of the stochastic noise makes manifest interference effects among the flow of modes reentering the horizon after inflation ends, and the superhorizon modes semiclassically produced at large scales by large space-time gradients, see e.g. [41,42,46,47]. The formula for the noise depends on the number of e-folds of cosmic expansion, and it rapidly approaches a constant value after a few e-folds. Also, we prove that our final results do not depend on the choice of infrared cutoff, the latter providing contributions that are exponentially suppressed by the number 
of e-folds of expansion. Our stochastic formalism can then be used to compute the spectrum of tensor fluctuations at horizon exit, that in appropriate limits coincide with the results of QFT calculations.

Our work aims to put in a firmer footing the intuitive idea that the stochastic distribution of tensor fields at superhorizon scales is due to the flow of modes between subhorizon and superhorizon scales. A general lesson of our approach is that a classical, stochastic approach to primordial tensor fluctuations from inflation is feasible and provides new physical insight in cosmological situations not usually considered in a stochastic context. Our results are consistent expectations from a traditional QFT approach to cosmological fluctuations from inflation. It can be used for better clarifying the classical dynamics of tensor modes at large superhorizon scales, and for dealing with large infrared effects from long wavelength modes. We summarize and further discuss physical implications of our results in section 4, which is followed by a technical appendix A. Throughout this work we set $\hbar=c=1$.

\section{A Fokker-Planck equation for tensor modes from inflation}

After crossing the cosmological horizon, single-field inflationary scalar and tensor fluctuations become time independent, and their spatial configurations can be described in terms of classical, but stochastically distributed superhorizon modes.

Our aim in this section is to discuss a systematic method for obtaining the classical evolution equation describing stochastic, coarse-grained superhorizon modes, starting from basic principles of quantum mechanics. We discuss free theories in arbitrary cosmological backgrounds equipped with a cosmological horizon, with the specific aim of extracting the effects of curved space on the derivation of the stochastic equation. We concentrate on tensor fluctuations, being the stochastic approach for scalar fluctuations already well developed (including the effects of self-interactions).

For determining the desired stochastic equation, we make use of the approach of $[27,29]$ based on the Schrödinger functional picture, first applied to inflationary cosmology in [45]. (See instead [48-50] for derivations of inflationary stochastic equations using a Schwinger-Keldish approach.) We start in subsection 2.1 setting the stage for the system we consider, and reviewing how the Schrödinger formalism leads to an evolution equation for probability densities associated with quantum Fourier modes of inflationary fluctuations. In subsection 2.2 we define the coarse-grained superhorizon quantities we consider, and we derive the classical stochastic evolution equation for the distribution of the coarse-grained quantities. The result is a Fokker-Planck evolution equation, for which we provide the expressions for noise and drift. In subsection 2.3 we discuss how to use these results for computing the spectrum of tensor fluctuations evaluated at horizon crossing, as well as the Gibbs entropy associated with superhorizon coarse-grained tensor modes.

\subsection{The system we consider}

We consider a space-time described by a conformally flat FRW metric perturbed by spin-2 tensor perturbations:

$$
d s^{2}=a^{2}(\tau)\left[-d \tau^{2}+\left[\delta_{i j}+h_{i j}(\tau, \vec{x})\right] d x^{i} d x^{j}\right],
$$


where $a(\tau)$ is the scale factor, while $h_{i j}$ denotes the linearized, transverse-traceless tensor fluctuation, gauge invariant at first order in perturbations. We do not need to specify the explicit time dependence of the scale factor for developing our arguments, which can then be applied to a variety of cosmological setups (see section 3 ). The effective quadratic action controlling the tensor modes in eq $(2.1)$ is

$$
S_{h}^{(2)}=\frac{M_{\mathrm{Pl}}^{2}}{8} \int d \tau d^{3} x a^{2}(\tau)\left[h_{i j}^{\prime 2}-\left(\vec{\nabla} h_{i j}\right)^{2}\right],
$$

where prime denotes derivative along conformal time.

We express tensor fluctuations in Fourier space, defined within a box of comoving size $L$ (in due time we will consider the limit of infinitely large box size):

$$
h_{i j}(\tau, \vec{x})=\frac{2}{M_{\mathrm{Pl}} L^{3}} \sum_{\lambda} \sum_{\vec{k}} h_{k}^{(\lambda)}(\tau) \mathbf{e}_{i j}^{(\lambda)}(\hat{k}) e^{i \vec{k} \vec{x}},
$$

with $\vec{k}=k \hat{k}$ the tensor 3-momentum, and $\lambda$ its polarization. $\mathbf{e}_{i j}^{( \pm)}(\hat{k})$ are (real) helicity tensors normalized as (we sum over repeated spatial indexes)

$$
\mathbf{e}_{i j}^{(\lambda)}(\hat{k}) \mathbf{e}_{i j}^{\left(\lambda^{\prime}\right)}(\hat{k})=2 \delta^{\lambda \lambda^{\prime}} .
$$

To ensure that $h_{i j}(\tau, \vec{x})$ is real, we demand $\left(h_{k}^{(\lambda)}(\tau)\right)^{*}=h_{-k}^{(\lambda)}(\tau)$, and in writing eq (2.3) we sum over positive as well as negative values of $k$. Plugging eq (2.3) in (2.2), we find the quadratic action for mode of momentum $k$ :

$$
S_{k}=\sum_{\lambda} \int d \tau a^{2}(\tau)\left[h_{k}^{(\lambda)} h_{-k}^{(\lambda)}-k^{2} h_{k}^{(\lambda)} h_{-k}^{(\lambda)}\right] .
$$

The associated Lagrangian density $\mathcal{L}_{k}$ is the argument of the previous integral, and allows us to define the momentum

$$
\pi_{k}^{(\lambda)} \equiv \frac{\delta \mathcal{L}_{k}}{\delta h_{k}^{\prime(\lambda)}}=a^{2}(\tau) h_{-k}^{(\lambda)}
$$

This information can be used to obtain the Hamiltonian density

$$
\mathcal{H}_{k}^{(\lambda)}=\frac{1}{a^{2}(\tau)} \pi_{k}^{(\lambda)} \pi_{-k}^{(\lambda)}+a^{2}(\tau) k^{2} h_{k}^{(\lambda)} h_{-k}^{(\lambda)}
$$

which is a basic ingredient for our next discussion.

\section{The functional Schrödinger picture}

We make use of the functional Schrödinger picture to derive the evolution equation for probability densities for the system under consideration. We apply the approach previously developed in [27] to the case of spin-2 tensor modes. In this subsection we make use of basic rules of quantum mechanics; in the next subsection 2.2 we show how an appropriate coarse-grained procedure leads to a classical, stochastic evolution equation for the superhorizon quantities we are interested in.

In the functional Schrödinger formalism, the quantities $h_{k}^{(\lambda)}$ and $\pi_{k}^{(\lambda)}$ are promoted to operators 
$\hat{h}_{k}^{(\lambda)}$ and $\hat{\pi}_{k}^{(\lambda)}$. An abstract quantum mechanical state in Fourier space is realized by $\Psi_{k}^{(\lambda)}\left(h_{k}^{(\lambda)}, \tau\right)$ which is a wave functional of the $c$-number quantity $h_{k}^{(\lambda)}$, and it is evaluated at a time $\tau$. The action of the operator $\hat{h}_{k}^{(\lambda)}$ on the quantum state is realized by multiplying $\Psi_{k}^{(\lambda)}$ by $h_{k}^{(\lambda)}$, while the action of the canonical momentum $\hat{\pi}_{k}^{(\lambda)}$ is realized by functional differentiation:

$$
\begin{aligned}
\hat{h}_{k}^{(\lambda)}\left|\Psi_{k}^{(\lambda)}\right\rangle & \rightarrow h_{k}^{(\lambda)} \Psi_{k}^{(\lambda)}, \\
\hat{\pi}_{k}^{(\lambda)}\left|\Psi_{k}^{(\lambda)}\right\rangle & \rightarrow \frac{1}{i} \frac{\partial \Psi_{k}^{(\lambda)}}{\partial h_{k}^{(\lambda)}} .
\end{aligned}
$$

The Schrödinger formalism dictates that for each mode $k$ and polarization $\lambda$ the evolution of the quantum state is controlled by the Schrödinger equation

$$
i \frac{\partial \Psi_{k}^{(\lambda)}(\tau)}{\partial \tau}=\mathcal{H}_{k}^{(\lambda)} \Psi_{k}^{(\lambda)}(\tau)
$$

with Hamiltonian

$$
\mathcal{H}_{k}^{(\lambda)}=-\frac{1}{a^{2}(\tau)} \frac{\delta^{2}}{\delta h_{k}^{(\lambda)} \delta h_{-k}^{(\lambda)}}+a^{2}(\tau) k^{2} h_{k}^{(\lambda)} h_{-k}^{(\lambda)} .
$$

Ours is a free theory, and we can use a Gaussian Ansatz for parametrizing the wave function. We assume no parity violation, hence the explicitly time-dependent functions appearing in our Ansatz are assumed not to depend on the polarization index $\lambda$ :

$$
\Psi_{k}^{(\lambda)}\left[h_{k}^{(\lambda)}, \tau\right]=\Omega_{k}(\tau) \exp \left\{-a^{2}(\tau)\left[\alpha_{k}(\tau) h_{k}^{(\lambda)} h_{-k}^{(\lambda)}-\beta_{0}(\tau) \delta_{k 0} h_{k}^{(\lambda)}\right]\right\}
$$

The zero-mode contribution proportional to $\beta_{0}$ is not forbidden hence we need to include it - as we will see it is relevant when discussing the effects of the zero mode of infinitely large wavelength.

Plugging Ansatz (2.12) in (2.10), the system of equations to solve is (all quantities a part from $k$ depend on time $\tau$ )

$$
\begin{aligned}
0 & =\Omega_{k}^{\prime}+i \alpha_{k} \Omega_{k}, \\
0 & =\alpha_{k}^{\prime}+i \alpha_{k}^{2}+\frac{2 a^{\prime}}{a} \alpha_{k}-i k^{2}, \\
0 & =\beta_{0}^{\prime}+i \alpha_{0} \beta_{0}+\frac{2 a^{\prime}}{a} \beta_{0} .
\end{aligned}
$$

Combining the last two equations, we find the relation $\beta_{0}(\tau)=\mathcal{C}_{\beta} \alpha_{0}(\tau)$, with $\mathcal{C}_{\beta}$ arbitrary constant (that will not enter in our final results). To deal with eq (2.14), it is convenient to define [45]

$$
\alpha_{k}(\tau)=\frac{1}{i} \partial_{\tau} \ln \left[\frac{\gamma_{k}^{\star}(\tau)}{a(\tau)}\right]
$$

Plugging in eq (2.14) we get a second order, linear equation for $\gamma_{k}$

$$
\gamma_{k}^{\prime \prime}+\left(k^{2}-\frac{a^{\prime \prime}}{a}\right) \gamma_{k}=0
$$


Since the definition (2.16) involves derivatives of a logarithm, we can choose the preferred normalization for the mode $\gamma_{k}$. We impose the Wronskian condition

$$
\gamma_{k}^{\prime} \gamma_{k}^{\star}-\gamma_{k}^{\prime \star} \gamma_{k}=i
$$

Given these conditions, the following relations hold

$$
\begin{aligned}
\alpha_{k}+\alpha_{k}^{\star} & =-\frac{1}{\left|\gamma_{k}\right|^{2}}, \\
\alpha_{k}-\alpha_{k}^{\star} & =\frac{1}{i} \partial_{\tau} \ln \left[\frac{\left|\gamma_{k}\right|^{2}}{a^{2}(\tau)}\right] .
\end{aligned}
$$

For any $k \neq 0$, we can impose the Bunch-Davies initial conditions at early times $\tau \rightarrow-\infty$, since at very small scales the effect of space-time curvature can be neglected. As shown in [45], this is equivalent to ensure that the wave function at early times is the one of a harmonic oscillator. These conditions completely fix the solution for each mode $k \neq 0$.

We need special care in dealing with the zero mode. In this case the Bunch-Davies condition does not apply, since $k=0$ can never acquire a small-scale limit for any given time $\tau$. The Fourier mode $k=0$ is a linear combination of the two independent solutions

$$
\gamma_{0}(\tau) \propto a(\tau), \gamma_{0}(\tau) \propto a(\tau) \mathcal{I}(\tau)
$$

which solve eq $(2.17)$. The quantity $\mathcal{I}(\tau)$ is defined as

$$
\mathcal{I}(\tau)=\int_{\tau_{\star}}^{\tau} \frac{d \tilde{\tau}}{a^{2}(\tilde{\tau})}
$$

with $\tau_{\star}$ an arbitrary fiducial time. We find convenient to express the zero mode as

$$
\frac{\gamma_{0}(\tau)}{a(\tau)}=\sqrt{\frac{1}{2 \mu \sin (\Delta \theta)}}+\sqrt{\frac{\mu e^{2 i \Delta \theta}}{2 \sin (\Delta \theta)}} \mathcal{I}(\tau),
$$

with $\Delta \theta$, and $\mu$ two arbitrary real quantities. Their values can be associated with the initial conditions on the zero mode at fiducial time $\tau_{*}$. We will study in what comes next how the dependence on initial conditions affects the structure of the evolution equations for the quantities we are interested in.

The expression (2.23) automatically satisfies the Wronskian condition. An overall phase can be included in the zero-mode solution, but it has no physical consequences. The solution for $\alpha_{0}$, as defined in (2.16), reads

$$
\alpha_{0}(\tau)=\frac{\mu e^{-i(\Delta \theta+\pi / 2)}}{a^{2}(\tau)} \frac{1}{1+\mu e^{-i \Delta \theta} \mathcal{I}(\tau)} .
$$

It depends on $\mu$ and $\Delta \theta$, determined by the initial conditions at time $\tau_{*}$. But notice that the value of $\alpha_{0}(\tau)$ depends also on the integral $\mathcal{I}(\tau)$ which depends on the entire cosmological history from the fiducial initial time $\tau_{\star}$ to $\tau$.

Once we have control on the quantities entering in the wave functional, we define a probability 
density associated with the quantum state of momentum $k$. As usual in quantum mechanics, this quantity is proportional to the square of the wave functional ${ }^{1}$ :

$$
P_{k}^{(\lambda)}=\left|\Psi_{k}^{(\lambda)}\right|^{2}
$$

Using the relation (2.12), the normalized probability reads

$$
P_{k}^{(\lambda)}=\frac{f_{k}}{\pi} \exp \left\{-f_{k}\left(h_{k}^{(\lambda)}-g_{0}^{\star} \frac{\delta_{k 0}}{f_{k}}\right)\left(h_{-k}^{(\lambda)}-g_{0} \frac{\delta_{k 0}}{f_{k}}\right)\right\}
$$

with

$$
\begin{aligned}
& f_{k}=a^{2}(\tau)\left(\alpha_{k}(\tau)+\alpha_{-k}(\tau)\right), \\
& g_{0}=a^{2}(\tau) \beta_{0}(\tau)=\mathcal{C}_{\beta} a^{2}(\tau) \alpha_{0}(\tau) .
\end{aligned}
$$

The probability density $P_{k}^{(\lambda)}$ is an important building block for the arguments we develop next. In fact, we will work only in terms of probabilities for determining our stochastic evolution equation. By differentiating along time, and by making use of the evolution equation (2.14), it is straightforward to prove that it satisfies a Fokker-Planck-like equation:

$$
\frac{\partial P_{k}^{(\lambda)}}{\partial \tau}=\omega_{k} \frac{\partial^{2} P_{k}^{(\lambda)}}{\partial h_{k}^{(\lambda)} \partial h_{-k}^{(\lambda)}}+\omega_{0}\left[\frac{\partial}{\partial h_{k}^{(\lambda)}}\left(h_{k}^{(\lambda)} P_{k}^{(\lambda)}\right)+\frac{\partial}{\partial h_{-k}^{(\lambda)}}\left(h_{-k}^{(\lambda)} P_{-k}^{(\lambda)}\right)\right],
$$

with

$$
\begin{aligned}
\omega_{k} & =\frac{i}{a^{2}(\tau)} \frac{\alpha_{k}-\alpha_{0}-\alpha_{k}^{\star}+\alpha_{0}^{\star}}{\alpha_{k}+\alpha_{k}^{\star}}, \\
\omega_{0} & =-i\left(\alpha_{0}-\alpha_{0}^{*}\right) .
\end{aligned}
$$

Using equations (2.16) we can also reexpress the previous formula as

$$
\begin{aligned}
& \omega_{k}=-\frac{\left|\gamma_{0}(\tau)\right|^{2}}{a^{2}(\tau)} \partial_{\tau}\left(\frac{\left|\gamma_{k}(\tau)\right|^{2}}{\left|\gamma_{0}(\tau)\right|^{2}}\right), \\
& \omega_{0}=-\partial_{\tau} \ln \left(\frac{\left|\gamma_{0}(\tau)\right|^{2}}{a^{2}(\tau)}\right) .
\end{aligned}
$$

This is our starting point for developing a coarse-graining procedure to describe the dynamics of superhorizon modes.

\subsection{Coarse-graining superhorizon tensor modes}

We now apply the previous formulas to the development of a convenient coarse-grained tensor field at superhorizon scales, and its corresponding stochastic evolution equation.

We start defining long-wavelength (time-independent) superhorizon fields as a sum over Fourier

\footnotetext{
${ }^{1}$ We are focusing on the diagonal elements of the density matrix; we do not consider off-diagonal elements, which can be relevant for example to investigate decoherence processes and quantum-to-classical transition. See e.g. [32-37,39,40].
} 
modes, with a cutoff controlled by the comoving horizon scale $k_{h}$ :

$$
h_{i j}(\vec{x})=\frac{2}{M_{\mathrm{Pl}} L^{3}} \sum_{\lambda} \sum_{\vec{k},|k|<k_{h}} h_{k}^{(\lambda)} \mathbf{e}_{i j}^{(\lambda)}(\hat{k}) e^{i \vec{k} \vec{x}},
$$

where the cutoff scale is (we call $\mathcal{H}=a^{\prime} / a^{2}$ )

$$
k_{h} \equiv \sigma a(\tau) \mathcal{H}(\tau)
$$

and $0 \leq \sigma \leq 1$ is a constant that quantifies what fraction of long wavelength modes we include in the coarse-graining procedure. We express the sum in (2.34) in terms of the time independent $c$-numbers $h_{k}^{(\lambda)}$ we used in the previous subsection for expressing the waveform $\Psi$. The coarse-grained quantity $h_{i j}$ in eq (2.34) is time-independent and we expect it to be stochastically distributed at superhorizon scales. Being built in terms of the abstract $c$-numbers $h_{k}^{(\lambda)}$, it does not spoil the isotropy of the underlying space-time geometry.

In fact, the coarse-grained quantity $h_{i j}$ of eq (2.34) is a natural definition of coarse-grained superhorizon tensor mode, and we use it in what follows. Being constituted by a combination of $h_{k}^{(\lambda)}$ modes at large scales $k<k_{h}$, we define the probability density related with the coarse-grained quantity $h_{i j}$ as the product of the independent probabilities associated with each of the Fourier modes entering in eq (2.34):

$$
P\left(\tau, h_{i j}(\vec{x})\right) \equiv \Pi_{\lambda} \Pi_{|k|<k_{h}} P_{k}^{(\lambda)} .
$$

Notice that the product depends only on the size of the momenta, and not on their directions. Starting from the $P_{k}^{(\lambda)}$ evolution equation (2.29) for any given mode $k$, it is straightforward to obtain an evolution equation for $P\left(\tau, h_{i j}\right)$. Selecting any given $k$, we first multiply both sides of (2.29) for all the remaining probability densities $\ldots P_{k-2}^{(\lambda)} P_{k-1}^{(\lambda)} P_{k+1}^{(\lambda)} \ldots$ Then, using eq $(2.36)$ and the Leibniz rule, we can reconstruct an equation for $P\left(\tau, h_{i j}(\vec{x})\right)$.

As shown in the technical appendix A, the final result is a Fokker-Planck equation controlling the probability density $P\left(\tau, h_{i j}\right)$

$$
\frac{1}{a(\tau) \mathcal{H}(\tau)} \frac{\partial P\left(\tau, h_{i j}\right)}{\partial \tau}=\mathcal{N}(\tau) \frac{\partial^{2} P\left(\tau, h_{i j}\right)}{\partial h_{i j}^{2}}+\mathcal{D}(\tau) \frac{\partial}{\partial h_{i j}}\left[h_{i j} P\left(\tau, h_{i j}\right)\right]
$$

The time derivative in the left-hand side is assembled for convenience in the combination

$$
a \mathcal{H} d \tau=H d t=d n,
$$

with $n$ the e-fold number

$$
n=\log \left(a / a_{*}\right),
$$

a quantity that physically makes manifest the universe rate of expansion, and that represents the physically correct time variable in the context of stochastic inflation [28]. 
The noise and drift in eq $(2.37)$ are given by ${ }^{2}$

$$
\begin{aligned}
\mathcal{N} & =\frac{2\left|\gamma_{0}(\tau)\right|^{2}}{M_{\mathrm{Pl}}^{2} \pi^{2} \mathcal{H}(\tau) a^{3}(\tau)} \int_{k_{h}}^{k_{s}} k^{2} d k \partial_{\tau}\left(\frac{\left|\gamma_{k}(\tau)\right|^{2}}{\left|\gamma_{0}(\tau)\right|^{2}}\right), \\
\mathcal{D} & =-\frac{2}{\mathcal{H}(\tau) a(\tau)} \partial_{\tau} \ln \left(\frac{\left|\gamma_{0}(\tau)\right|^{2}}{a^{2}(\tau)}\right) .
\end{aligned}
$$

Notice that while the drift depends on the zero mode only, the noise involves an integration over all the super-horizon modes, with an horizon-size lower cutoff given by $k_{h}=\sigma a \mathcal{H}$ as in eq (2.35), and an upper cutoff $k_{s}$ which controls the total size of the superhorizon region experienced by the long modes. As we will see, the final results do not depend on $k_{s}$, at least for physically relevant scenarios, hence there are no large infrared effects depending on the total size of the superhorizon region. In fact, we can continuously reduce the size of $k_{s}$ in the final results, making it as small as we please.

Up to an irrelevant constant overall factor, we can write

$$
\begin{aligned}
\left|\gamma_{0}(\tau)\right|^{2} & \propto a^{2}(\tau)\{1+\sigma \mathcal{I}(\tau)[2 \cos \Delta \theta+\sigma \mathcal{I}(\tau)]\} \\
& =a^{2}(\tau)\{1+\sigma \Pi(\tau)\}
\end{aligned}
$$

where $\mathcal{I}(\tau)=\int_{\tau_{\star}}^{\tau} d \tilde{\tau} / a^{2}(\tilde{\tau})$ as given in eq $(2.22)$. We introduce

$$
\Pi(\tau) \equiv 2 \mathcal{I}(\tau) \cos \Delta \theta+\mu \mathcal{I}^{2}(\tau),
$$

with $\mu$ the constant parameter appearing in the solution for the zero mode, see eq (2.24). Substituting these expressions in formulas (2.40) and (2.41) we get

$$
\begin{aligned}
\mathcal{N} & =\frac{2(1+\mu \Pi(\tau))}{M_{\mathrm{Pl}}^{2} \pi^{2} \mathcal{H}(\tau) a(\tau)} \int_{k_{h}}^{k_{s}} k^{2} d k \partial_{\tau}\left(\frac{\left|\gamma_{k}(\tau)\right|^{2}}{a^{2}(\tau)(1+\mu \Pi(\tau))}\right) \\
\mathcal{D} & =\frac{2}{\mathcal{H}(\tau) a(\tau)} \partial_{\tau} \ln \left(\frac{1}{1+\mu \Pi(\tau)}\right) .
\end{aligned}
$$

It is also interesting to explicitly consider cases where the decaying mode contribution is set to zero, by selecting $\mu=0$. Then the drift vanishes, $\mathcal{D}=0$, since eq. (2.46) is proportional to $\mu$. The noise instead simplifies to

$$
\mathcal{N}=\frac{2}{\pi^{2} M_{\mathrm{Pl}}^{2} \mathcal{H}(\tau) a(\tau)} \int_{k_{h}}^{k_{s}} k^{2} d k \partial_{\tau}\left(\frac{\left|\gamma_{k}(\tau)\right|^{2}}{a^{2}(\tau)}\right)
$$

a formula that plays an important role for our applications. The following physically relevant properties are worth emphasizing:

- Expressions (2.37), (2.45) and (2.46) are general and valid for any cosmological space-time $a(\tau)$. Once we have control on the expressions for $\gamma_{k}(\tau)$ for each $k$, we can compute - analytically or

\footnotetext{
${ }^{2}$ We pass to the continuous limit taking a large size $L$, and expressing the sum as an integral: $\left(1 / L^{3}\right) \sum_{k}=$ $1 /(2 \pi)^{3} \int d^{3} k$.
} 
numerically - the expressions for noise and drift (2.45), (2.46) in a broad variety of physically interesting situations.

- The noise $\mathcal{N}$ in eq (2.45) is controlled by a sum of time derivatives of superhorizon modes, and depends on the time dependence of all the superhorizon modes $k_{s} \leq k \leq k_{h}$.

As we will see in the next section, such time dependence is a feature of rapidly expanding space-times, and the integral (2.45) is associated with the rate of change of the comoving horizon. This phenomenon controls the flow of modes crossing the horizon, and fits well with the heuristic picture that a source for the stochastic noise $\mathcal{N}$ is due to modes continuously crossing the cosmological horizon separating large and small scales. Effectively, we are dealing with an open system [27], and the flow of modes produces an analog of Brownian motion at cosmological scales.

Importantly, the order of integration in eq $(2.45)$ is from $k_{h}$ to $k_{s}$, and physically assumes that the noise is due to the flow of modes crossing the horizon from subhorizon to superhorizon scales. In a case where the situation is reversed, as what happens during standard cosmological epochs after inflation ends, the order of integration should be reversed for obtaining a noise with positive sign (see examples in section 3 ).

Also, eq (2.45) can include additional sources of noise in the superhorizon regime, due to correlations among positive and negative frequency modes with the same momentum $k$. As an example, noise can be generated by particle production at superhorizon scales after the transition between distinct cosmological space-times, as what happens between inflation and radiation domination. As far as we are aware, this is the first time these phenomena are explored in the context of a stochastic approach to tensor fluctuations. We will discuss explicit examples of these possibilities in section 3 .

- The drift term (2.46) depends on the physics of the zero mode $\gamma_{0}$ : the decaying mode appearing in $\gamma_{0}$ introduces a dependence on initial conditions at early times, through the coefficients of the integral $\mathcal{I}(\tau)$. On the other hand, if the cosmological evolution corresponds to an attractor, $\mathcal{I}$ and $\Pi$ become rapidly a constant: all the effects of the decaying mode drop out from expressions (2.45) and (2.46), and the dynamics is well described by Markovian evolution, independent from initial conditions. In this limit (or alternatively switching off the effects of the decaying mode by selecting $\mu=0$ ) the drift vanishes, and the noise reduces to eq (2.47).

\subsection{The spectrum and entropy of inflationary tensor modes}

\section{The spectrum}

In many cosmological situations it is important to compute the spectrum of tensor modes at horizon crossing. It is straightforward to obtain its expression starting from the Fokker-Planck equation we derived. For simplicity we consider an attractor cosmological evolution, where the decaying mode becomes rapidly negligible, and the drift vanishes. The Fokker-Planck equation (2.37) corresponds to Einstein formulation of the theory of Brownian motion, and reads (we express it in terms of the 
e-fold number, $d n=a \mathcal{H} d \tau)$

$$
\frac{\partial P}{\partial n}=\mathcal{N}(n) \frac{\partial^{2} P}{\partial h_{i j}^{2}} .
$$

This equation can be easily integrated providing the Gaussian probability density, when assuming a positive $\mathcal{N}$

$$
P\left(n, h_{i j}\right)=\frac{1}{\sqrt{2 \pi B(n)}} e^{-\frac{h_{i j}^{2}}{2 B(n)}} \quad, \quad \text { with } B^{\prime}(n)=2 \mathcal{N}(n) .
$$

Such probability density leads to the two-point function for superhorizon tensor modes as

$$
\left\langle h_{i j}^{2}(\tau, x)\right\rangle=\int d h_{i j} h_{i j}^{2} P=B(n) .
$$

The two-point function depends on the e-fold number, and it is independent from the spatial position. The same quantity $\left\langle h_{i j}^{2}\right\rangle$ can also be expressed in Fourier space, as an integral over the long wavelength tensor spectrum up to the cutoff scale:

$$
\left\langle h_{i j}^{2}(\tau, x)\right\rangle=\int_{\ln k_{s}}^{\ln k_{h}} \mathcal{P}_{T} d \ln k .
$$

To compute the value of the tensor spectrum at horizon scales we can use equations (2.50) and (2.51) together, as discussed in [51], and use $d \ln k_{h}=d \ln (a \mathcal{H})$.

We obtain

$$
\begin{aligned}
\mathcal{P}_{T} & =\frac{d\left\langle h_{i j}^{2}(\tau, x)\right\rangle}{d \ln k_{h}} \\
& =\left(\frac{d n}{d \ln (a \mathcal{H})}\right) \frac{d\left\langle h_{i j}^{2}(\tau, x)\right\rangle}{d n}=\left(\frac{d n}{d \ln (a \mathcal{H})}\right) \frac{d B(n)}{d n} \\
& =\frac{2 a^{2} \mathcal{H}^{2}}{\left|a^{2} \mathcal{H}^{2}+a \mathcal{H}^{\prime}\right|} \mathcal{N} .
\end{aligned}
$$

Hence ${ }^{3}$, knowing the profile of $\mathcal{N}$ as a function of the e-fold number $n$, equation (2.52) provides the tensor spectrum at horizon crossing. For the three cases of pure de Sitter, radiation domination, and matter domination that we study next we find

$$
\frac{2 a^{2} \mathcal{H}^{2}}{\left|a^{2} \mathcal{H}^{2}+a \mathcal{H}^{\prime}\right|}= \begin{cases}2 & \text { for de Sitter } \\ 2 & \text { for radiation domination } \\ 4 & \text { for matter domination }\end{cases}
$$

It is interesting to compare it with the tensor spectrum deep at superhorizon scales, computed with

\footnotetext{
${ }^{3}$ In cosmological phases following the end of inflation the denominator of the overall coefficient in eq (2.52) would be negative, in absence of the absolute value. However, in these cases the flow of modes is from superhorizon to subhorizon scales (see comment in the second bullet point after eq (2.47)). This fact changes our arguments here by an overall sign, leading to expression in eq (2.52) (with the absolute value).
} 
standard QFT methods (see e.g. [52]). One gets

$$
\mathcal{P}_{T}=\lim _{k \rightarrow 0} \frac{4 k^{3}}{\pi^{2}} \frac{\left|\gamma_{k}\right|^{2}}{a^{2}},
$$

where the modes $\gamma_{k}$ are solutions of eq (2.17). Notice that while eq (2.54) depends only on very large-scale modes with $k \rightarrow 0$, the stochastic prediction (2.52) depends on the noise $\mathcal{N}$ which involves a combination over all the superhorizon modes.

\section{The Gibbs entropy}

It is also interesting to compute the classical Gibbs entropy associated with our coarse-grained definition of superhorizon tensor fluctuations, see eq (2.34). (See also [53] for a discussion on the entropy of tensor fluctuations from inflation.) From the expression (2.49) (we set to one the Boltzmann constant), we get

$$
\begin{aligned}
S(n) & =-\int d h_{i j} P\left(n, h_{i j}\right) \ln \left[P\left(n, h_{i j}\right)\right], \\
& =\frac{1}{2} \ln [B(n)]+\text { constant }
\end{aligned}
$$

We learn that the entropy increases with the universe expansion, as long as the noise is positive:

$$
\frac{d S}{d n}=\frac{B^{\prime}(n)}{2 B(n)}=\frac{1}{2} \frac{\mathcal{N}(n)}{\int^{n} \mathcal{N}\left(n^{\prime}\right) d n^{\prime}}
$$

If the noise $\mathcal{N}$ is constant (or if it rapidly approaches a constant), then $B \propto 2 n$, and we find that the rate of variation of the entropy is inversely proportional to the e-fold number: $d S / d n=1 /(2 n)$. In our set up the Gibbs entropy grows logarithmically with the number of e-folds $n: S \propto(\log n) / 2$.

Let us briefly discuss the conceptually important case of de Sitter space, and compare our coarse grained entropy with the Gibbons-Hawking entropy $S_{d S}=\pi M_{\mathrm{Pl}}^{2} / H_{0}^{2}$ (with $H_{0}$ the constant Hubble parameter). As we are going to learn in section 3, the noise is constant in de Sitter, hence the coarse grained entropy associated with superhorizon tensor modes grows logarithmically as $S_{c g}=\log \sqrt{n / n_{\star}}$, with $n_{\star}$ a reference e-fold number. $S_{c g}$ contributes to the energy budget, and keeps smaller than $S_{d S}$ as long as $n \leq e^{2 S_{d S}}$, a limit on the e-fold number imposed by the entropy bound. We point out however that here we only considered the coarse-grained Gibbs entropy, while we do not include entanglement effects that have been argued to contribute to the entropy budget by a function linearly growing with the number of e-folds: see [37].

\section{Applications}

The general formulas we obtained in the previous section will now be applied to physically interesting cases, also in contexts that are seldom considered in stochastic approaches to cosmological inflation.

In section 3.1 we use our stochastic approach to reproduce in this context well-known QFT results for the spectrum of superhorizon tensor modes during inflation. We also go beyond the standard case, including in our stochastic approach a scenario with a phase of non-attractor evolution. 
In section 3.2 we consider the case of power-law inflation controlled by a parameter $\epsilon$ controlling the departure from a pure de Sitter expansion. We show that our formalism is sufficiently flexible to provide an exact, analytic expression for the noise that reduces to the de Sitter one in the limit $\epsilon \rightarrow 0$. We also show that the tilt $n_{T}$ of the tensor spectrum obtained by our stochastic method satisfies the expected consistency relation $n_{T}=-2 \epsilon$. We derive an expression for the tensor spectrum using the stochastic formulation that does not require $\epsilon$ to be small.

In section 3.3 we then consider cosmological scenarios where epochs of radiation and matter domination follow the phase of inflation. In this situation, we are interested to derive a stochastic formulation able to describe superhorizon tensor modes in the process of reentering the horizon after inflation ends. We find that this flow of modes from large towards small scales can be influenced by those genuinely superhorizon modes created by space-time curvature during radiation and matter dominated eras. We derive the corresponding expressions for the tensor spectrum at horizon exit, and compute the associated coarse-grained Gibbs entropy.

\subsection{Pure de Sitter expansion (plus an extension to non-attractor evolution)}

We start discussing the simplest case of a de Sitter universe, described by the conformal scale factor

$$
a=-\frac{1}{H_{0} \tau},
$$

with $\tau<0$, and $H_{0}$ a constant of dimensions of inverse time, corresponding to the Hubble parameter $H(\tau)=a^{\prime} / a^{2}$. For this choice of the scale factor, one has

$$
\frac{a^{\prime \prime}}{a}=\frac{2}{\tau^{2}}
$$

The mode function solving eq (2.17), satisfying the Bunch-Davies conditions, results

$$
\gamma_{k}=\frac{1}{\sqrt{2 k}} e^{-i k \tau} \times\left(1-\frac{i}{k \tau}\right)
$$

up to an overall phase that does not enter into the final results. The integral $\mathcal{I}(\tau)$ of eq $(2.22)$ controlling the effect of the decaying mode is $\left(\tau_{\star} \leq \tau \leq 0\right)$

$$
\begin{aligned}
\mathcal{I}(\tau) & =\int_{\tau_{\star}}^{\tau} d \tau^{\prime} H_{0}^{2} \tau^{\prime 2}=\frac{H_{0}^{2}}{2}\left(\tau^{3}-\tau_{\star}^{3}\right), \\
& =-\frac{H_{0}^{2} \tau_{\star}^{3}}{2}\left(1-e^{-3 n}\right),
\end{aligned}
$$

where the number $n$ of e-folds is defined as $n=\ln \left[a(\tau) / a\left(\tau_{\star}\right)\right]$. This implies that the quantity $\mathcal{I}$ rapidly approaches a constant during inflation, and its contributions to noise and drift are exponentially suppressed: we can safely assume that contributions proportional to $\sigma$ vanish in all our expressions. Hence the drift contribution to the Fokker-Planck equation is zero in this limit. 
For computing the noise we need the combination

$$
\begin{aligned}
\frac{\left|\gamma_{k}\right|^{2}}{a^{2}} & =\frac{H_{0}^{2}}{2 k^{3}}\left(1+k^{2} \tau^{2}\right)=\frac{H_{0}^{2}}{2 k^{3}}\left(1+\frac{k^{2}}{H_{0}^{2} a^{2}}\right), \\
& =\frac{H_{0}^{2}}{2 k^{3}}\left(1+\frac{k^{2}}{H_{0}^{2} a_{\star}^{2}} e^{-2 n}\right) .
\end{aligned}
$$

Its time (or e-fold) dependence - which controls the noise, see eq (2.47) - is limited to the second term inside the parentheses, characterizing the rate of change of the comoving horizon.

Substituting eq (3.6) in the expression (2.47) for the noise, we can easily perform the integral. We get the expression

$$
\mathcal{N}=\frac{H_{0}^{2}}{M_{\mathrm{Pl}}^{2} \pi^{2}}\left(\sigma^{2}-k_{s}^{2} \tau_{\star}^{2} e^{-2 n}\right) .
$$

The result depends on the choice of the cutoff $k_{h}=\sigma a \mathcal{H}$, and the infrared cutoff $k_{s}$. For the cutoff $k_{h}$ we choose $\sigma=1$ : we include all the super-horizon modes starting from horizon crossing, assuming all of them contribute in forming the noise. The choice of the infrared cutoff $k_{s}$ is instead not important, since its contribution is exponentially suppressed as the e-fold number increases. After a few e-folds we then get the following expression:

$$
\mathcal{N}=\frac{H_{0}^{2}}{M_{\mathrm{Pl}}^{2} \pi^{2}} .
$$

This is the expected result for the noise coefficient. Indeed, using the fact that $d n=d \ln a \mathcal{H}$ for a pure de Sitter evolution, eq (2.52) provides

$$
\mathcal{P}_{T}=\frac{2 H_{0}^{2}}{\pi^{2} M_{\mathrm{Pl}}^{2}},
$$

which is the well-known spectrum of tensor modes at very large scales in the limit of pure de Sitter expansion, obtained using QFT methods and formula (2.54). The statistics of the stochastic spectrum of coarse-grained modes maintains its properties from horizon exit up to very large scales, as expected given that the influence of decaying modes is negligible.

We now briefly discuss how these classic results can change, modifying one of the assumptions made so far for the case of pure de Sitter expansion. During inflation, transitory phases of nonattractor can enhance the spectrum of fluctuations - this mechanism is particularly interesting in view of producing primordial black holes (see e.g. $[54,55]$ for reviews). While this possibility has been mostly explored in the scalar sector, it might occur in the tensor sector as well $[56,57]$. During non-attractor, the would-be decaying mode proportional to the quantity $\int^{\tau} d \tilde{\tau} / a^{2}(\tilde{\tau})$ does not decay but grows. Possible effects of this phenomenon for what respects quantum contributions to stochastic quantities have been explored in recent literature, see e.g. [58-62].

Here we focus our analysis on understanding how a non-attractor regime influences the classical drift in the stochastic Fokker-Planck equation, using the formalism we developed.

The simplest possibility to consider is a model of non-attractor corresponding to a contracting universe, with $a(\tau)=a_{0} \tau^{2}$ ( $a_{0}$ is a normalization factor, and $-\infty<\tau<0$ ) so that $a^{\prime \prime} / a=2 / \tau^{2}$, 
as for the case of de Sitter (see eq (3.2)). This implies that the solution for the mode function $\gamma_{k}$ is the same as in de Sitter expansion. The number $n$ of e-folds of contraction is connected to the time variable by $\tau=\tau_{*} e^{-2 n}$, for $\tau_{*}<\tau<0$.

We find that the integral $\mathcal{I}$ is

$$
\mathcal{I}=-\frac{1}{3 a_{0}^{2} \tau_{\star}^{3}}\left(e^{3 n / 2}-1\right)
$$

so it exponentially grows with the number $n$ of e-folds of contraction (instead of approaching a constant as in de Sitter, see eq (3.5)). Calculating the drift as in eq (2.46), in the limit of large e-fold number we find the expression

$$
\mathcal{D}=-6-6 e^{-3 n / 2}\left(1+\frac{3 a_{0}^{2} \tau_{\star}^{3} \cos \Delta}{\mu}\right)+\mathcal{O}\left(e^{-3 n}\right) .
$$

So the drift approaches an order-one constant as contraction proceeds, and can influence considerably the stochastic evolution. It would be interesting to study more generally stochastic features of non-attractor inflation using our method: we postpone this investigation to future analysis.

\subsection{Power-law expansion}

We now apply our formalism to power-law expansion, described by the scale factor

$$
a(\tau)=-\frac{1}{H_{0} \tau^{1 /(1-\epsilon)}},
$$

for constant $\epsilon$, with de Sitter space corresponding to $\epsilon=0$. The parameter $\epsilon$ is associated with derivatives of the Hubble parameter $\mathcal{H}=(d a) /\left(a^{2} d \tau\right)$ through the definition

$$
\epsilon=-\frac{1}{\mathcal{H}^{2}} \frac{d \mathcal{H}}{a d \tau} .
$$

We are interested here in cosmological space-times with $0 \leq \epsilon<1$. We can then express the Hubble parameter and second time derivative of the scale factor as

$$
\begin{aligned}
a \mathcal{H} & =-\frac{1}{(1-\epsilon) \tau}, \\
\frac{a^{\prime \prime}}{a} & =\frac{1-\epsilon / 2}{(1-\epsilon)^{2}} \frac{2}{\tau^{2}} .
\end{aligned}
$$

It is a textbook exercise to obtain the solution for the mode functions that approaches a BunchDavies vacuum at early times - see e.g. [52]. From such a solution one gets $\left(H_{\nu}^{(1)}(y)\right.$ as the Hankel function of the first kind)

$$
\begin{aligned}
\frac{\left|\gamma_{k}\right|^{2}}{a^{2}(\tau)} & =\frac{\pi(-k \tau)}{2 k a^{2}(\tau)}\left|H_{\nu}^{(1)}(-k \tau)\right|^{2} \\
& =\frac{\pi H_{0}^{2}}{2} \frac{(-k \tau)^{(3-\epsilon) /(1-\epsilon)}}{k^{(3-\epsilon) /(1-\epsilon)}}\left|H_{\nu}^{(1)}(-k \tau)\right|^{2}
\end{aligned}
$$


where we denote

$$
\nu=\frac{3}{2} \frac{1-\epsilon / 3}{1-\epsilon} .
$$

For calculating the noise, we assume that the UV cutoff is $k_{h}=a \mathcal{H}$, selecting $\sigma=1$ in eq (2.35): in other words, as in section 3.1 we include all super-horizon modes in our definition of coarse-grained tensor quantity. The value of $k_{s}$ is not important, since its contributions to the integral exponentially decay to zero as a function of the e-fold number: in what follows for simplicity we set $k_{s}=0$. The noise is, always assuming $\epsilon<1$,

$$
\begin{aligned}
\mathcal{N} & =\frac{2}{M_{\mathrm{Pl}}^{2} \pi^{2} \mathcal{H}(\tau) a(\tau)} \int_{a \mathcal{H}}^{0} k^{2} d k \partial_{\tau}\left(\frac{\left|\gamma_{k}(\tau)\right|^{2}}{a^{2}(\tau)}\right) \\
& =\frac{H_{0}^{2}}{2 \pi M_{\mathrm{Pl}}^{2}}(1-\epsilon)(-\tau)^{\frac{2 \epsilon}{1-\epsilon}} \int_{0}^{1-\epsilon} d(-k \tau)(-k \tau)^{\frac{-2 \epsilon}{1-\epsilon}} \frac{d}{d(-k \tau)}\left((-k \tau)^{\frac{3-\epsilon}{1-\epsilon}}\left|H_{\nu}^{(1)}(-k \tau)\right|^{2}\right) \\
& =\frac{2 H_{0}^{2} G(\epsilon)}{\pi^{2} M_{\mathrm{Pl}}^{2}}(-\tau)^{\frac{2 \epsilon}{1-\epsilon}}
\end{aligned}
$$

where the overall coefficient $G(\epsilon)$ is given by

$$
G(\epsilon)=\pi(1-\epsilon) \int_{0}^{1 /(1-\epsilon)} x^{3} d x\left[J_{\frac{1+\epsilon}{2-\epsilon}}(x) J_{\frac{3-\epsilon}{2-\epsilon}}(x)+Y_{\frac{1+\epsilon}{2-\epsilon}}(x) Y_{\frac{3-\epsilon}{2-\epsilon}}(x)\right]
$$

with $J_{\nu}(x), Y_{\nu}(x)$ denoting respectively Bessel functions of the first and second kind. The function $G(\epsilon)$ tends to 1 for $\epsilon$ small:

$$
G(\epsilon) \simeq 1+2.94 \epsilon+\mathcal{O}\left(\epsilon^{2}\right)
$$

and is represented in Fig 1.

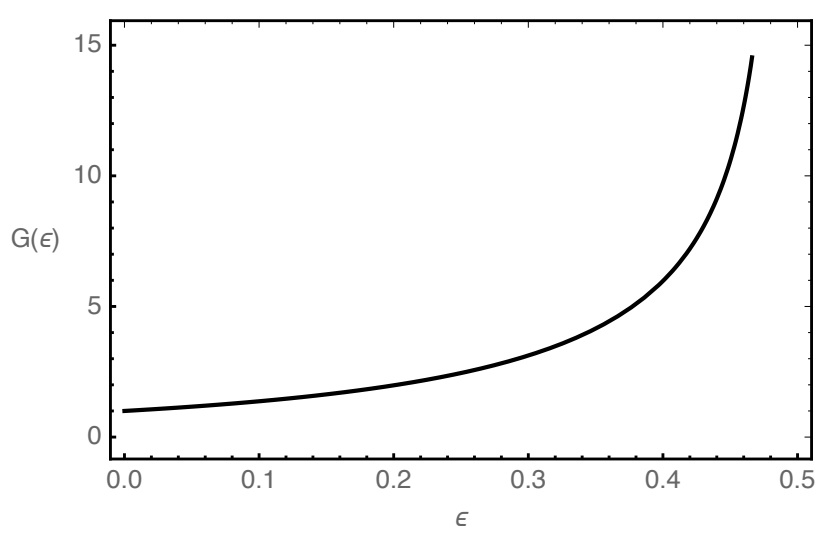

Figure 1: Plot of the function $G(\epsilon)$ given in eq (3.21) as a function of $\epsilon$.

Since in this cosmological era the number of e-folds is connected to time by ( $\tau_{*}$ being a fiducial time $\left.\tau_{\star} \leq \tau \leq 0\right)$

$$
\tau=\tau_{\star} e^{-n(1-\epsilon)}
$$


We can then write the expression for the noise (choosing for definiteness $\tau_{\star}=-1$ )

$$
\mathcal{N}=\frac{H_{0}^{2} G(\epsilon)}{4 \pi^{2}} e^{-2 \epsilon n}
$$

The corresponding tensor spectrum is given by formula (2.52). Its tilt satisfies the well-known relation

$$
n_{T}=\frac{d \ln \mathcal{P}_{T}}{d \ln k}=\frac{d \ln \mathcal{N}}{d n}=-2 \epsilon,
$$

in agreement with standard QFT methods.

\subsection{From inflation to radiation and to matter domination}

After inflation ends, the standard picture of big bang cosmology starts, and the universe enters in a phase of radiation followed by matter domination. Inflationary superhorizon modes reenter the horizon during these phases, and begin evolving and propagating through cosmological distances. During radiation or matter domination, the stochastic distribution of superhorizon modes can be described in terms of the physical arguments we developed in the previous sections. The time-varying size of the cosmological horizon leads to a flow of modes back from superhorizon to subhorizon scales - a process contributing to the stochastic noise in the Fokker-Planck equation for our coarse-grained quantity. In fact, we have an open system where the Brownian motionlike phenomenon is induced by the 'holes' left by the modes that leave the superhorizon regime. Moreover, the transition from inflation to radiation domination leads to particle production at super-horizon scales, see e.g. [41, 42, 46, 47], and [52] for a textbook discussion. We might suspect that the superhorizon stochastic distribution gets affected by such phenomena.

In order to describe an universe where inflation (approximated as de Sitter space) is followed by radiation domination, we parametrize the scale factor as

$$
\begin{aligned}
& a(\tau)=-\frac{1}{H_{0}\left(\tau-\tau_{0}\right)} \quad \tau<0, \\
& a(\tau)=\frac{\tau+\tau_{0}}{H_{0} \tau_{0}^{2}} \quad \tau>0,
\end{aligned}
$$

for a continuous transition among the two regimes at $\tau=0\left(\tau_{0}>0\right.$ is a fiducial time $)$. During radiation domination, the solution for mode function $\gamma_{k}$ is a linear combination of plane waves

$$
\gamma_{k}=c_{1}(k) e^{i k \tau}+c_{2}(k) e^{-i k \tau} \quad \tau>0,
$$

whose scale-dependent coefficients are determined by the Israel conditions with inflationary modes in Bunch-Davies vacuum at $\tau<0$

$$
\begin{aligned}
c_{1} & =\frac{e^{i k \tau_{0}}}{\sqrt{8 k^{5}} \tau_{0}^{2}}, \\
c_{2} & =-\frac{e^{i k \tau_{0}}}{\sqrt{8 k^{5}} \tau_{0}^{2}}\left(1-2 i k \tau_{0}-2 k^{2} \tau_{0}^{2}\right)
\end{aligned}
$$


We call negative frequency modes (in analogy with their Minkowski counterparts) the terms weighted by $c_{2}(k)$ in eq (3.28). Their contribution leads to particle production and amplification of of particle number at superhorizon scales. We can compute the quantity entering in the noise integrand in eq (2.47). We get for $\tau>0$

$$
\frac{\left|\gamma_{k}\right|^{2}}{a^{2}(\tau)}=\frac{H_{0}^{2}}{4 k^{5}\left(\tau+\tau_{0}\right)^{2}}\left[1+2 k^{4} \tau_{0}^{4}+2 k \tau_{0} \sin (2 k \tau)-\left(1-2 k^{2} \tau^{2}\right) \cos (2 k \tau)\right] .
$$

The oscillatory contributions within the parentheses are due to interferences between positive and negative frequency modes with the same $k$. Starting from expression (3.31), using the definition in eq (2.54), it is straightforward to compute the spectrum of tensor fluctuations at late times $\tau / \tau_{0} \gg 1$. We obtain $[46]$

$$
\mathcal{P}_{T}=\frac{2 H_{0}^{2}}{\pi^{2} M_{\mathrm{Pl}}^{2}}\left(\frac{\sin k \tau}{k \tau}\right)^{2},
$$

at very large scales, $k \tau \ll 1$, we find $\mathcal{P}_{T}=2 H_{0}^{2} /\left(\pi^{2} M_{\mathrm{Pl}}^{2}\right)$.

We now analyze the problem from the perspective of the stochastic formalism developed in the previous sections. The number of e-folds from the onset of radiation domination is

$$
\frac{\tau}{\tau_{0}}=e^{n}-1
$$

The integral $\mathcal{I}$ of eq $(2.22)$ results

$$
\begin{aligned}
\mathcal{I}(n) & =\int_{\tau_{0}}^{\tau} \frac{d \tilde{\tau}}{a^{2}(\tilde{\tau})}, \\
& =\tau_{0}^{3} H_{0}^{2}\left(1-e^{-n}\right),
\end{aligned}
$$

hence for increasing $n$ it approaches a constant, although more slowly than in de Sitter space: the drift and the effects of the zero mode nevertheless are suppressed after a few e-folds, and we neglect them. Starting from eq (3.31), it is straightforward to perform the analytic integrations ${ }^{4}$ associated with the noise of eq (2.47). A mixing between positive and negative frequency modes of momentum $k$ is induced by the square of the mode function $\left|\gamma_{k}\right|^{2} / a^{2}(\tau)$, and leads to interesting effects.

The noise $\mathcal{N}$ controlling superhorizon modes during radiation domination is computed by the integral in eq (2.47), choosing $k_{h}=\sigma a \mathcal{H}$, and leaving an arbitrary small $k_{s}$ as infrared cut-off. The quantity $\mathcal{N}(n)$ written as a function of the e-fold number results

$$
\begin{aligned}
\mathcal{N}(n)= & \frac{H_{0}^{2}}{\pi^{2} M_{\mathrm{Pl}}^{2}} \frac{\sin ^{2} \sigma}{\sigma^{2}} \\
\times & \left\{1-\frac{H_{0}^{2} \sigma^{2} e^{-2 n}}{2 k_{s}^{2} \sin ^{2} \sigma}\left[1-2 k_{s}^{4} \tau_{0}^{4}-\left(1-2 k_{s}^{2} \tau_{0}^{2}\right) \cos \left(2 k_{s} \tau_{0}\left(1-\frac{e^{n}}{H_{0} \tau_{0}}\right)\right)\right.\right. \\
& \left.\left.\quad-2 k_{s} \tau_{0} \sin \left(2 k_{s} \tau_{0}\left(1-\frac{e^{n}}{H_{0} \tau_{0}}\right)\right)\right]\right\}+\mathcal{O}\left(e^{-3 n}\right),
\end{aligned}
$$

\footnotetext{
${ }^{4}$ Recall that we are in a situation where the flow of modes is from superhorizon to subhorizon scales, hence we should place an overall minus sign in eq (2.47), as explained in the second point after that formula.
} 

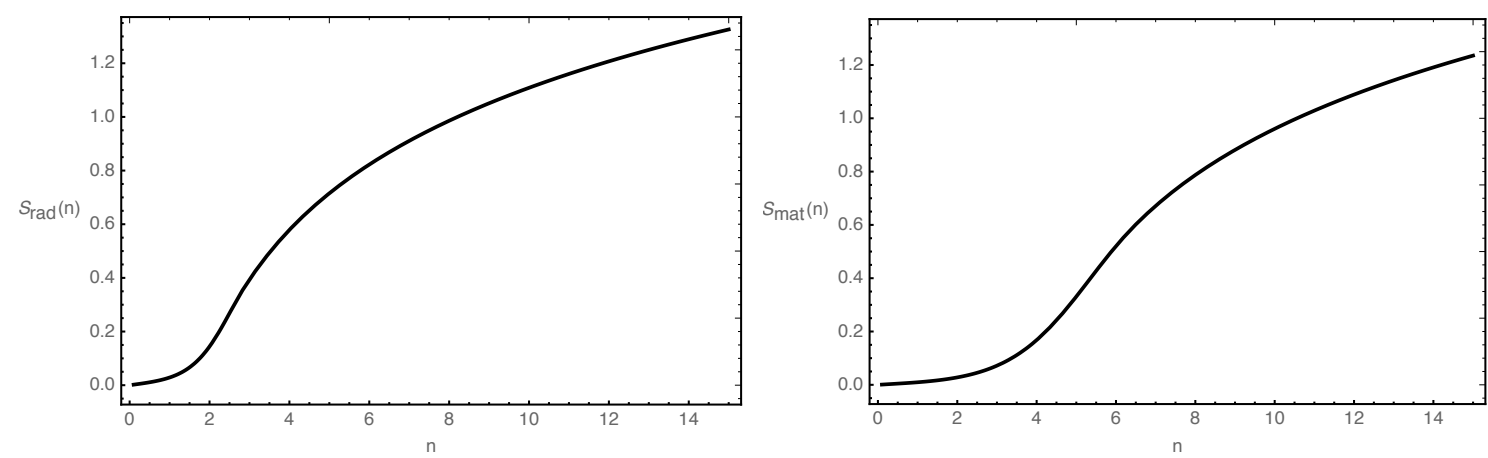

Figure 2: Plot of the coarse-grained entropy of superhorizon tensor modes as a function of the e-fold number. We apply eq (2.56) to epochs of radiation and matter domination. The integration constant of eq (2.56) has been chosen in such a way that the entropy vanishes at $n=0$. We choose $H_{0} \tau_{0}=4$, and $k_{s} \tau_{0}=0.1, \sigma=0.02$. In both cases the entropy scales as $\ln \left(n^{1 / 2}\right)$ for large $n$.

where the quantity $\mathcal{O}\left(e^{-3 n}\right)$ decays at least as fast as $e^{-3 n}$ with the number of e-folds of evolution. We checked that the complete expression for the noise is continuous when sending $k_{s} \rightarrow 0$. Notice that the explicit dependence on $k_{s}$ of eq (3.36) is exponentially suppressed with the e-fold number: after a few e-folds, the noise approaches a constant given by

$$
\mathcal{N}(n \gg 1)=\frac{H_{0}^{2}}{\pi^{2} M_{\mathrm{Pl}}^{2}} \frac{\sin ^{2} \sigma}{\sigma^{2}} .
$$

Using eqs (2.52), (2.53), we find for the tensor spectrum at horizon crossing, evaluated after few e-folds of expansion, results

$$
\mathcal{P}_{T}(n \gg 1)=\frac{2 H_{0}^{2}}{\pi^{2} M_{\mathrm{Pl}}^{2}} \frac{\sin ^{2} \sigma}{\sigma^{2}} .
$$

When $\sigma \ll 1$, the quantity in eq (3.38) coincides with the QFT tensor spectrum of eq (3.32) when evaluated deep at superhorizon scales $k \tau \rightarrow 0$. Physically, this choice for $\sigma$ implies that we include in the coarse-graining procedure only modes at very large scales, well beyond the horizon size - see our definition of UV cut-off in eq (2.35). This result is intuitively clear since the QFT approach focuses precisely on modes at very large scales, hence there is no surprise that in this limit the two approaches agree. Spanning the value of $\sigma$ within the interval $0 \leq \sigma \leq 1$, the size of the spectrum reduces of around $70 \%$ with respect to its $\sigma=0$ value. We interpret this suppression as due to interference effects among modes spontaneously created by space-time gradients at superhorizon scales, and modes flowing from super to subhorizon scales. Such interference is reduced when coarse graining only over modes deep in the superhorizon regime, $\sigma \rightarrow 0$.

We can also consider the case where a phase of matter domination follows the radiation-dominated era considered above. The scale factors in the three epochs read $\left(\tau_{0}>0\right.$ and $\left.\tau_{b}>0\right)$

$$
a(\tau)= \begin{cases}-\frac{1}{H_{0}\left(\tau-\tau_{0}\right)} & \text { for } \tau \leq-\tau_{b}, \\ \frac{\tau+\tau_{0}+2 \tau_{b}}{H_{0}\left(\tau_{b}+\tau_{0}\right)^{2}} & \text { for }-\tau_{b} \leq \tau \leq 0, \\ \frac{\left(\tau+2 \tau_{0}+4 \tau_{b}\right)^{2}}{4 H_{0}\left(\tau_{b}+\tau_{0}\right)^{2}\left(\tau_{0}+2 \tau_{b}\right)} & \text { for } \tau \geq 0\end{cases}
$$


and are continuous with their first derivative continuous at the transition epochs. In the limit of very short radiation-dominated era, $\tau_{b} / \tau_{0} \ll 1$, the solution for the mode function in matter domination for $\tau \geq 0$ reads

$$
\gamma_{k}=\frac{3}{8 \sqrt{2}} \frac{e^{-i k\left(\tau+\tau_{0}\right)}}{\tau_{0}^{3} k^{9 / 2}} \frac{\left(i+k \tau_{0}\right)\left(-i+k \tau+2 k \tau_{0}\right)}{\tau+2 \tau_{0}} .
$$

Proceeding as above in the radiation-dominated case, we find the following expression for the noise as function of e-folds in the matter-dominated era, $n \geq 0$

$$
\begin{aligned}
\mathcal{N}(n)= & \frac{9 H_{0}^{2}}{128 \pi^{2} M_{\mathrm{Pl}}^{2}} \frac{(\sin (2 \sigma)-2 \sigma \cos (2 \sigma))^{2}}{\sigma^{6}} \\
& +\frac{9 H_{0}^{2}}{512 \sigma^{4} \pi^{2} M_{\mathrm{Pl}}^{2}} e^{-n}\left[3+16 \sigma^{2}+\left(8 \sigma^{2}-3\right) \cos (4 \sigma)-12 \sigma \sin (4 \sigma)\right] \\
& +\mathcal{O}\left(e^{-2 n}\right) .
\end{aligned}
$$

After few e-folds of matted-dominated expansion, the noise approaches a constant. In this limit, using eqs (2.52) and (2.53), we find the tensor spectrum at horizon crossing

$$
\mathcal{P}_{T}=\frac{18 H_{0}^{2}}{\pi^{2} M_{\mathrm{Pl}}^{2}} \frac{(\sin (2 \sigma)-2 \sigma \cos (2 \sigma))^{2}}{(2 \sigma)^{6}},
$$

which approaches the standard large-scale value $\mathcal{P}_{T}=2 H_{0}^{2} /\left(\pi^{2} M_{\mathrm{Pl}}^{2}\right)$ in the limit $\sigma \ll 1$. As for the case of radiation domination, the limit of small- $\sigma$ implies the inclusion only of very large-scale modes in the coarse-graining procedure. In spanning through the interval $0<\sigma<1, \mathcal{P}_{T}$ monotonically decreases, reducing to a size of $43 \%$ with respect to the $\sigma=0$ value.

We conclude with few words about the behaviour of the coarse-grained Gibbs entropy, as derived in eq (2.56). Both for the cases of radiation and matter domination the noise approaches a constant as the cosmological evolution proceeds, and the number of e-folds increases. In the transition between inflation and matter domination the noise has a richer profile: we use it for plotting the expression of the entropy in Fig 2. We notice that in both cases the entropy increases as function of the e-fold number, with a steep slope for $n$ between 2 and 6 . Then, for large $n$, the entropy scales as $\ln \left(n^{1 / 2}\right)$, as expected.

\section{Conclusions}

We discussed a coarse-grained prescription for describing the stochastic superhorizon dynamics of inflationary tensor modes, which seed the spectrum of primordial gravitational waves from inflation. We made precise the intuitive idea that the stochastic distribution of tensor fields at superhorizon scales is due to the flow of tensor modes between subhorizon and superhorizon scales. Our aim was to put in a firm footing a consistent description of inflationary tensor modes which allows one to deal with large infrared effects that characterize the dynamics of light fields in inflation.

Using basic principles of quantum mechanics, we showed how the probability density for the coarse-grained tensor modes satisfies a stochastic Fokker-Planck equation, whose noise and drift are computable and depend on the cosmological system under consideration. The evolution is well 
described by a standard Markovian process if the cosmological expansion follows an attractor, and we also considered how the dynamics is affected by the presence of non-attractor eras. Our stochastic formulas are applied to a variety of cosmological frameworks, also cases not often considered in the context of stochastic inflation. We obtained the expected results for noise and drift in pure de Sitter and power-law inflation. But we also explored consequences of non-attractor phases as for example a contracting universe. Most notably, we considered a cosmological space-time with transition from de Sitter (inflationary) phase to radiation and to matter domination. This is the first time this topic is discussed in the context of a stochastic approach to superhorizon tensor modes. The computation of the stochastic noise made manifest interference effects among the flow of modes reentering the horizon after inflation ends, and the superhorizon modes semiclassically produced at large scales by large space-time gradients. The formula for the noise depends on the number of e-folds of cosmic evolution, and it rapidly approaches a constant value after few e-folds of expansion. We proved that our final results do not depend on the choice of infrared cutoff. Our stochastic results are then compared with the standard predictions of QFT applied to cosmology. The two approaches give the same results for the power spectrum of tensor fluctuations if the coarse-graining procedure includes only modes deep in the superhorizon regime. This fact is intuitively clear since the QFT approach focusses precisely on modes at very large scales. We also quantitatively computed the effect of including a larger portion of superhorizon modes in the coarse-graining prescription, showing that it can change the amplitude of the tensor spectrum at horizon crossing by an overall numerical coefficient of order one. Hence, depending on the prescription adopted, the predictions for the amplitude of the spectrum can change. It would be interesting to further consolidate our physical understanding of this fact, and its phenomenological consequences.

This work contains various novel results both for developing a stochastic approach to tensor fields from inflation, and for applying it to a variety of cosmological settings. It would be interesting to further develop this approach to better understand how much our quantitative results for noise and drift depend on the detailed features of cosmological space-times, and on the transitions among different cosmological eras. It would also be interesting to include self-interactions (cubic or higher) among tensor fluctuations, and then also include the effects of scalar perturbations in the analysis.

\section{Acknowledgments}

It is a pleasure to thank Cliff Burgess and Ivonne Zavala for useful comments. GT is partially supported by the STFC grant ST/T000813/1.

\section{A Fokker-Planck equation and coarse graining}

In this appendix we show how to make use of our coarse-graining procedure to pass from the evolution equation (2.29) for a single-mode $k$ to the coarse-grained Fokker-Planck equation eq (2.37). The idea is to multiply both sides of (2.29) - defined for a certain fiducial mode $k$ - for all the remaining probability densities $\ldots P_{k-2}^{(\lambda)} P_{k-1}^{(\lambda)} P_{k+1}^{(\lambda)} \ldots$ of the remaining modes. Then, using the definition of eq $(2.36)$, we reconstruct an evolution equation for $P\left(\tau, h_{i j}(\vec{x})\right)$. To do so, we we also 
need the fact that

$$
\frac{\partial h_{i j}(\vec{x})}{\partial h_{k}^{(\lambda)}}=\frac{2 e^{i \vec{k} \vec{x}} \mathbf{e}_{i j}^{(\lambda)}(\hat{k})}{M_{\mathrm{Pl}} L^{3 / 2}} \quad ; \quad \frac{\partial h_{i j}(\vec{x})}{\partial h_{-k}^{(\lambda)}}=\frac{2 e^{-i \vec{k} \vec{x}} \mathbf{e}_{i j}^{(\lambda)}(\hat{k})}{M_{\mathrm{Pl}} L^{3 / 2}}
$$

We proceed to discuss this procedure analyzing its consequences for each term of eq (2.29).

i) The time derivative in the left-hand-side (LHS). We multiply the LHS of eq (2.29) by all the the $P_{k^{\prime}}^{(\lambda)}$ with $k^{\prime} \neq k$. We sum over momenta (positive and negative) and polarizations, obtaining

$$
\sum_{\lambda} \sum_{k} \ldots P_{k-1}^{(-\lambda)} P_{k-1}^{(\lambda)} P_{k}^{(-\lambda)} \frac{\partial P_{k}^{(\lambda)}}{\partial \tau} P_{k+1}^{(\lambda)} P_{k+1}^{(-\lambda)} \ldots=\frac{\partial P}{\partial \tau} .
$$

ii) The first derivatives in the right-hand-side (RHS). We multiply the RHS of eq (2.29) by all the $P_{k^{\prime}}^{(\lambda)}$ with $k^{\prime} \neq k$. We sum over momenta (positive and negative) and polarizations, obtaining

$$
\begin{aligned}
& \sum_{\lambda} \sum_{k} \ldots P_{k-1}^{(-\lambda)} P_{k-1}^{(\lambda)} \omega_{0}\left[h_{k}^{(\lambda)} \frac{\partial}{\partial h_{k}^{(\lambda)}}\left(P_{k}^{(\lambda)}\right)\right] P_{k}^{(-\lambda)} P_{k+1}^{(\lambda)} P_{k+1}^{(-\lambda)} \ldots= \\
= & \omega_{0} \sum_{\lambda} \sum_{k}\left[h_{k}^{(\lambda)} \frac{\partial P}{\partial h_{k}^{(\lambda)}}\right], \\
= & \omega_{0} \sum_{\lambda} \sum_{k}\left[h_{k}^{(\lambda)} \frac{\partial h_{i j}}{\partial h_{k}^{(\lambda)}}\right] \frac{\partial P}{\partial h_{i j}}, \\
= & \omega_{0} h_{i j} \frac{\partial P}{\partial h_{i j}},
\end{aligned}
$$

since we recall $\omega_{0}$ is independent from $k$. In the previous expression, we sum over indexes $i j$.

iii) The second derivatives in the RHS. We proceed as before, and we express it as

$$
\begin{aligned}
& \sum_{\lambda} \sum_{k} \ldots P_{k-1}^{(-\lambda)} P_{k-1}^{(\lambda)} \omega_{k}\left[\frac{\partial^{2} P_{k}^{(\lambda)}}{\partial h_{k}^{(\lambda)} \partial h_{-k}^{(\lambda)}}\right] P_{k}^{(-\lambda)} P_{k+1}^{(\lambda)} P_{k+1}^{(-\lambda)} \ldots= \\
= & \sum_{\lambda} \sum_{k} \omega_{k} \frac{\partial^{2} P}{\partial h_{k}^{(\lambda)} \partial h_{-k}^{(\lambda)}}, \\
= & \sum_{\lambda} \sum_{k} \omega_{k} \frac{\partial h_{i j}}{\partial h_{k}^{(\lambda)}} \frac{\partial h_{i j}}{\partial h_{-k}^{(\lambda)}} \frac{\partial^{2} P}{\partial h_{i j}^{2}}, \\
= & \left(\sum_{k} \omega_{k}\right)\left(\sum_{\lambda} \mathbf{e}_{i j}^{(\lambda)} \mathbf{e}_{i j}^{(\lambda)}\right) \frac{\partial^{2} P}{\partial h_{i j}^{2}}, \\
= & 2\left(\sum_{k} \omega_{k}\right) \frac{\partial^{2} P}{\partial h_{i j}^{2}} .
\end{aligned}
$$


Collecting the results we just obtained, we can write a stochastic Fokker-Planck evolution equation for the coarse-grained probability $P$, which reads

$$
\frac{1}{a \mathcal{H}} \frac{\partial P}{\partial \tau}=\mathcal{N} \frac{\partial^{2} P}{\partial h_{i j}^{2}}+\mathcal{D} \frac{\partial}{\partial h_{i j}}\left(h_{i j} P\right),
$$

and noise and drift given in the main text: see eqs (2.45) and (2.46).

\section{References}

[1] A. H. Guth, "The Inflationary Universe: A Possible Solution to the Horizon and Flatness Problems," Phys. Rev. D 23 (1981) 347-356.

[2] A. Albrecht and P. J. Steinhardt, "Cosmology for Grand Unified Theories with Radiatively Induced Symmetry Breaking," Phys. Rev. Lett. 48 (1982) 1220-1223.

[3] A. D. Linde, "A New Inflationary Universe Scenario: A Possible Solution of the Horizon, Flatness, Homogeneity, Isotropy and Primordial Monopole Problems," Phys. Lett. B 108 (1982) 389-393.

[4] V. F. Mukhanov and G. V. Chibisov, "Quantum Fluctuations and a Nonsingular Universe," JETP Lett. 33 (1981) 532-535.

[5] A. H. Guth and S. Y. Pi, "Fluctuations in the New Inflationary Universe," Phys. Rev. Lett. 49 (1982) 1110-1113.

[6] S. W. Hawking, "The Development of Irregularities in a Single Bubble Inflationary Universe," Phys. Lett. B 115 (1982) 295.

[7] A. A. Starobinsky, "Dynamics of Phase Transition in the New Inflationary Universe Scenario and Generation of Perturbations," Phys. Lett. B 117 (1982) 175-178.

[8] L. H. Ford, "Quantum Instability of De Sitter Space-time," Phys. Rev. D 31 (1985) 710.

[9] I. Antoniadis, J. Iliopoulos, and T. N. Tomaras, "Quantum Instability of De Sitter Space," Phys. Rev. Lett. 56 (1986) 1319.

[10] N. C. Tsamis and R. P. Woodard, "Strong infrared effects in quantum gravity," Annals Phys. 238 (1995) 1-82.

[11] Y. Urakawa and T. Tanaka, "Influence on Observation from IR Divergence during Inflation. I.," Prog. Theor. Phys. 122 (2009) 779-803, arXiv:0902.3209 [hep-th].

[12] S. B. Giddings and M. S. Sloth, "Semiclassical relations and IR effects in de Sitter and slow-roll space-times," JCAP 01 (2011) 023, arXiv:1005.1056 [hep-th].

[13] C. T. Byrnes, M. Gerstenlauer, A. Hebecker, S. Nurmi, and G. Tasinato, "Inflationary Infrared Divergences: Geometry of the Reheating Surface versus $\delta N$ Formalism," JCAP 08 (2010) 006, arXiv:1005.3307 [hep-th]. 
[14] C. P. Burgess, R. Holman, L. Leblond, and S. Shandera, "Breakdown of Semiclassical Methods in de Sitter Space," JCAP 10 (2010) 017, arXiv:1005.3551 [hep-th].

[15] M. Gerstenlauer, A. Hebecker, and G. Tasinato, "Inflationary Correlation Functions without Infrared Divergences," JCAP 06 (2011) 021, arXiv:1102.0560 [astro-ph.CO].

[16] T. Tanaka and Y. Urakawa, "Large gauge transformation, Soft theorem, and Infrared divergence in inflationary spacetime," JHEP 10 (2017) 127, arXiv:1707.05485 [hep-th].

[17] A. A. Starobinsky, "STOCHASTIC DE SITTER (INFLATIONARY) STAGE IN THE EARLY UNIVERSE," Lect. Notes Phys. 246 (1986) 107-126.

[18] Y. Nambu and M. Sasaki, "Stochastic Stage of an Inflationary Universe Model," Phys. Lett. B 205 (1988) 441-446.

[19] H. E. Kandrup, "STOCHASTIC INFLATION AS A TIME DEPENDENT RANDOM WALK," Phys. Rev. D 39 (1989) 2245.

[20] Y. Nambu, "Stochastic Dynamics of an Inflationary Model and Initial Distribution of Universes," Prog. Theor. Phys. 81 (1989) 1037.

[21] S. Mollerach, S. Matarrese, A. Ortolan, and F. Lucchin, "Stochastic inflation in a simple two field model," Phys. Rev. D 44 (1991) 1670-1679.

[22] A. D. Linde, D. A. Linde, and A. Mezhlumian, "From the Big Bang theory to the theory of a stationary universe," Phys. Rev. D 49 1783-1826, arXiv:gr-qc/9306035.

[23] A. A. Starobinsky and J. Yokoyama, "Equilibrium state of a selfinteracting scalar field in the De Sitter background," Phys. Rev. D 50 6357-6368, arXiv:astro-ph/9407016.

[24] D. Wands, K. A. Malik, D. H. Lyth, and A. R. Liddle, "A New approach to the evolution of cosmological perturbations on large scales," Phys. Rev. D 62 (2000) 043527, arXiv:astro-ph/0003278.

[25] F. Finelli, G. Marozzi, A. A. Starobinsky, G. P. Vacca, and G. Venturi, "Generation of fluctuations during inflation: Comparison of stochastic and field-theoretic approaches," Phys. Rev. D 79 (2009) 044007, arXiv:0808.1786 [hep-th].

[26] B. Garbrecht, G. Rigopoulos, and Y. Zhu, "Infrared correlations in de Sitter space: Field theoretic versus stochastic approach," Phys. Rev. D 89 (2014) 063506, arXiv:1310.0367 [hep-th].

[27] C. P. Burgess, R. Holman, G. Tasinato, and M. Williams, "EFT Beyond the Horizon: Stochastic Inflation and How Primordial Quantum Fluctuations Go Classical," JHEP 03 (2015) 090, arXiv:1408.5002 [hep-th].

[28] V. Vennin and A. A. Starobinsky, "Correlation Functions in Stochastic Inflation," Eur. Phys. J. C75 (2015) 413, arXiv:1506.04732 [hep-th]. 
[29] C. P. Burgess, R. Holman, and G. Tasinato, "Open EFTs, IR effects \\& late-time resummations: systematic corrections in stochastic inflation," JHEP 01 (2016) 153, arXiv: 1512.00169 [gr-qc].

[30] T. J. Hollowood and J. I. McDonald, "Decoherence, discord and the quantum master equation for cosmological perturbations," Phys. Rev. D 95 no. 10, (2017) 103521, arXiv:1701.02235 [gr-qc].

[31] C. Pattison, V. Vennin, H. Assadullahi, and D. Wands, "Stochastic inflation beyond slow roll," JCAP 07 (2019) 031, arXiv:1905.06300 [astro-ph.CO].

[32] R. H. Brandenberger, R. Laflamme, and M. Mijic, "Classical Perturbations From Decoherence of Quantum Fluctuations in the Inflationary Universe," Mod. Phys. Lett. A 5 (1990) 2311-2318.

[33] A. Albrecht, P. Ferreira, M. Joyce, and T. Prokopec, "Inflation and squeezed quantum states," Phys. Rev. D 50 (1994) 4807-4820, arXiv:astro-ph/9303001.

[34] D. Polarski and A. A. Starobinsky, "Semiclassicality and decoherence of cosmological perturbations," Class. Quant. Grav. 13 (1996) 377-392, arXiv:gr-qc/9504030.

[35] E. Calzetta and B. L. Hu, "Quantum fluctuations, decoherence of the mean field, and structure formation in the early universe," Phys. Rev. D 52 (1995) 6770-6788, arXiv:gr-qc/9505046.

[36] J. Lesgourgues, D. Polarski, and A. A. Starobinsky, "Quantum to classical transition of cosmological perturbations for nonvacuum initial states," Nucl. Phys. B 497 (1997) 479-510, arXiv: gr-qc/9611019.

[37] C. Kiefer, D. Polarski, and A. A. Starobinsky, "Quantum to classical transition for fluctuations in the early universe," Int. J. Mod. Phys. D 7 (1998) 455-462, arXiv:gr-qc/9802003.

[38] F. C. Lombardo and D. Lopez Nacir, "Decoherence during inflation: The Generation of classical inhomogeneities," Phys. Rev. D 72 (2005) 063506, arXiv:gr-qc/0506051.

[39] C. P. Burgess, R. Holman, and D. Hoover, "Decoherence of inflationary primordial fluctuations," Phys. Rev. D 77 (2008) 063534, arXiv:astro-ph/0601646.

[40] C. Kiefer and D. Polarski, "Why do cosmological perturbations look classical to us?," Adv. Sci. Lett. 2 (2009) 164-173, arXiv:0810.0087 [astro-ph].

[41] L. P. Grishchuk, "Amplification of gravitational waves in an istropic universe," Zh. Eksp. Teor. Fiz. 67 (1974) 825-838.

[42] A. A. Starobinsky, "Spectrum of relict gravitational radiation and the early state of the universe," JETP Lett. 30 (1979) 682-685.

[43] A. A. Starobinsky, "A New Type of Isotropic Cosmological Models Without Singularity," Phys. Lett. B 91 (1980) 99-102. 
[44] L. P. Grishchuk and Y. V. Sidorov, "Squeezed quantum states of relic gravitons and primordial density fluctuations," Phys. Rev. D 42 (1990) 3413-3421.

[45] A. H. Guth and S.-Y. Pi, "The Quantum Mechanics of the Scalar Field in the New Inflationary Universe," Phys. Rev. D32 (1985) 1899-1920.

[46] L. F. Abbott and D. D. Harari, "Graviton Production in Inflationary Cosmology," Nucl. Phys. B 264 (1986) 487-492.

[47] L. H. Ford, "Gravitational Particle Creation and Inflation," Phys. Rev. D 35 (1987) 2955.

[48] M. Morikawa, "Dissipation and Fluctuation of Quantum Fields in Expanding Universes," Phys. Rev. D 42 (1990) 1027-1034.

[49] B. L. Hu and S. Sinha, "A Fluctuation - dissipation relation for semiclassical cosmology," Phys. Rev. D 51 (1995) 1587-1606, arXiv:gr-qc/9403054.

[50] S. Matarrese, M. A. Musso, and A. Riotto, "Influence of superhorizon scales on cosmological observables generated during inflation," JCAP 05 (2004) 008, arXiv:hep-th/0311059.

[51] K. E. Kunze, "Perturbations in stochastic inflation," JCAP 0607 (2006) 014, arXiv:astro-ph/0603575 [astro-ph].

[52] M. Maggiore, Gravitational Waves. Vol. 2: Astrophysics and Cosmology. Oxford University Press, 2018.

[53] C. Kiefer, D. Polarski, and A. A. Starobinsky, "Entropy of gravitons produced in the early universe," Phys. Rev. D 62 (2000) 043518, arXiv:gr-qc/9910065.

[54] B. Carr, F. Kuhnel, and M. Sandstad, "Primordial Black Holes as Dark Matter," Phys. Rev. D 94 no. 8, (2016) 083504, arXiv:1607.06077 [astro-ph.CO].

[55] M. Sasaki, T. Suyama, T. Tanaka, and S. Yokoyama, "Primordial black holes-perspectives in gravitational wave astronomy," Class. Quant. Grav. 35 no. 6, (2018) 063001, arXiv:1801.05235 [astro-ph.CO].

[56] M. Mylova, O. Özsoy, S. Parameswaran, G. Tasinato, and I. Zavala, "A new mechanism to enhance primordial tensor fluctuations in single field inflation," JCAP 12 (2018) 024, arXiv:1808.10475 [gr-qc].

[57] O. Ozsoy, M. Mylova, S. Parameswaran, C. Powell, G. Tasinato, and I. Zavala, "Squeezed tensor non-Gaussianity in non-attractor inflation," JCAP 09 (2019) 036, arXiv: 1902.04976 [hep-th].

[58] M. Biagetti, G. Franciolini, A. Kehagias, and A. Riotto, "Primordial Black Holes from Inflation and Quantum Diffusion," JCAP 07 (2018) 032, arXiv:1804.07124 [astro-ph.CO].

[59] J. M. Ezquiaga and J. García-Bellido, "Quantum diffusion beyond slow-roll: implications for primordial black-hole production," JCAP 08 (2018) 018, arXiv:1805.06731 [astro-ph.C0]. 
[60] D. Cruces, C. Germani, and T. Prokopec, "Failure of the stochastic approach to inflation beyond slow-roll," JCAP 03 (2019) 048, arXiv: 1807.09057 [gr-qc].

[61] J. M. Ezquiaga, J. García-Bellido, and V. Vennin, "The exponential tail of inflationary fluctuations: consequences for primordial black holes," JCAP 03 (2020) 029, arXiv:1912.05399 [astro-ph.CO].

[62] D. G. Figueroa, S. Raatikainen, S. Rasanen and E. Tomberg, "Non-Gaussian Tail of the Curvature Perturbation in Stochastic Ultraslow-Roll Inflation: Implications for Primordial Black Hole Production," Phys. Rev. Lett. 127 (2021) no.10, 101302, arXiv:2012.06551 [astro-ph.CO]. 